\title{
Dithering in Quantized RSS Based Localization
}

\author{
Di Jin, Feng Yin, Carsten Fritsche, Fredrik Gustafsson and Abdelhak M. Zoubir
}

\section{Linköping University Post Print}

\section{Tweet}

N.B.: When citing this work, cite the original article.

(C)2015 IEEE. Personal use of this material is permitted. However, permission to reprint/republish this material for advertising or promotional purposes or for creating new collective works for resale or redistribution to servers or lists, or to reuse any copyrighted component of this work in other works must be obtained from the IEEE.

Di Jin, Feng Yin, Carsten Fritsche, Fredrik Gustafsson and Abdelhak M. Zoubir, Dithering in Quantized RSS Based Localization, 2015, Proc. IEEE 6th Int. Workshop on Computational Advances in Multi-Sensor Adaptive Processing (CAMSAP).

Postprint available at: Linköping University Electronic Press

http://urn.kb.se/resolve?urn=urn:nbn:se:liu:diva-123709 


\section{Dithering in Quantized RSS Based Localization}

\author{
Di Jin and Abdelhak M. Zoubir \\ Signal Processing Group \\ Technische Universität Darmstadt \\ Darmstadt, Germany \\ \{djin, zoubir\}@spg.tu-darmstadt.de
}

\author{
Feng Yin \\ Ericsson $\mathrm{AB}$ \\ Linköping, Sweden \\ feng.yin@ericsson.com
}

\author{
Carsten Fritsche and Fredrik Gustafsson \\ Division of Automatic Control \\ Linköping University \\ Linköping, Sweden \\ \{carsten, fredrik\}@isy.liu.se
}

\begin{abstract}
We study maximum likelihood (ML) position estimation using quantized received signal strength measurements. In order to mitigate the undesired quantization effect in the observations, the dithering technique is adopted. Various dither noise distributions are considered and the corresponding likelihood functions are derived. Simulation results show that the proposed ML estimator with dithering is able to generate a significantly reduced bias but a modestly increased mean-squareerror as compared to the conventional ML estimator without dithering.
\end{abstract}

Keywords-Dithering, maximum likelihood estimation, localization, quantized received signal strength.

\section{INTRODUCTION}

Position information is crucial to various wireless sensor network (WSN) applications. Among different types of position-related measurements, received signal strength (RSS) is easy to obtain without dedicated hardware. Hence, the resulting localization system is often low cost, less complex, and easy to integrate into other systems. However, the uncertainty in RSS measurements about the position is usually larger than, for instance, time of arrival measured by ultrawideband systems [1]. In reality, quantization introduces additional uncertainty in the RSS observations. In the existing Wi-Fi network and bluetooth low energy (BLE) network, RSS is represented in the system using 6-8 bits (also known as the received signal strength indication (RSSI)). In some other networks with cheap sensors, even less bits might be used to represent a quantized RSS due to rather limited sensor readings. Since the release of the iBeacon protocol, there is a big trend to use only one bit quantized RSS, also known as proximity, for Geofencing and particle filtering [2]. The use of proximity measurements for localization is beneficial from various aspects. Among other advantages, the signaling between the user equipment and the core network can be significantly reduced by sending a binary value instead of a 6-8 bits RSSI value. As trade-off, localization using coarsely quantized RSS becomes more difficult.

The problem of parameter estimation based on quantized measurements has been addressed in various papers [3]-[7]. It is known from [8] that quantization introduces two kinds of errors - a first order effect, i.e., the quantization bias, and a higher order effect, i.e., aliasing. Adding proper dither noise, enables the reconstruction of the moments and even the distribution function of the non-quantized signal [9], and eventually reduces the estimation bias. However, the estimation variance may increase as a side-effect. The concept of using dither noise for improved performance is not new; One excellent example is the use of dithering in particle filters [10]. In this paper, we integrate the concept of dithering into the canonical maximumlikelihood (ML) framework for position estimation based on quantized RSS measurements. The influence of dithering on the performance of the ML estimator (MLE) is investigated.

Our contributions are as follows. First, we propose an MLE with dithering, aiming to mitigate the undesired quantization effect. Second, we list three commonly used dither noise distributions and derive the likelihood functions correspondingly. Third, we evaluate the proposed MLE with dithering and compare it with the one without dithering via simulations.

The remainder of this paper is organized as follows. Section II introduces the signal model. Section III revisits several commonly used dither noise distributions and derives the MLE for quantized and dithered RSS measurements. In Section IV, we present simulation results. Finally, Section V concludes the paper.

\section{Signal Model}

We consider a WSN consisting of $N$ anchors with known positions and a stationary agent with an unknown position in a two-dimensional (2-D) space. Let $\mathbf{x}=[x, y]^{T}$ be the position of the agent and $\mathbf{x}_{i}=\left[x_{i}, y_{i}\right]^{T}$ be the position of anchor $i, i=1,2, \ldots, N$. We consider one RSS measurement per anchor. We adopt the commonly used log-distance pathloss model and represent the continuous-valued RSS measurement from anchor $i$ as

$$
\begin{aligned}
y_{i} & =g_{i}(\mathbf{x})+n_{i}, \\
g_{i}(\mathbf{x}) & \triangleq A_{0}-10 n_{p} \log _{10}\left(d_{i}(\mathbf{x}) / d_{0}\right),
\end{aligned}
$$

where $A_{0}$ denotes the received power at a predefined reference distance $d_{0}, n_{p}$ denotes the propagation pathloss exponent, $d_{i}(\mathbf{x}) \triangleq\left\|\mathbf{x}_{i}-\mathbf{x}\right\|_{2}$ is the Euclidean distance between anchor $i$ and the agent, and the error terms $n_{i} \sim \mathcal{N}\left(0, \sigma_{i}^{2}\right)$, $i=1,2, \ldots, N$, account for the propagation shadowing effect and they are assumed to be mutually independent.

Figure 1 illustrates our signal model. In the non-dithered case, the quantized RSS measurement $r_{i}$ is obtained by quantizing $y_{i}$ according to,

$$
r_{i}=Q\left(y_{i}\right)=\left\{\begin{array}{cc}
1 & \text { if } P_{1} \leq y_{i}<P_{2} \\
2 & \text { if } P_{2} \leq y_{i}<P_{3} \\
\vdots & \vdots \\
S & \text { if } P_{S} \leq y_{i}<P_{S+1}
\end{array}\right.
$$

where $Q(\cdot)$ stands for a quantization operator and $P_{1}, P_{2}, \cdots, P_{S+1}$ are the quantization levels with $P_{1}=-\infty$, $P_{S+1}=+\infty$. Quantization level optimization is out of 


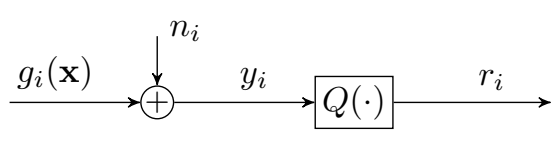

(a)

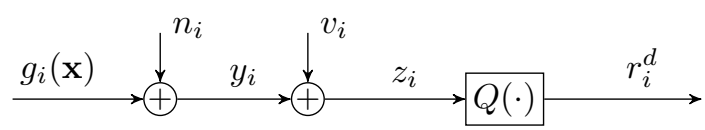

(b)

Fig. 1: The signal model of the quantized measurement from anchor $i$ for (a) non-dithered case and (b) dithered case.

the scope of this paper. Herein, we simply assume that the quantization levels have been selected a priori. In the dithered case, an independent dither noise variable $v_{i}$ is added to the quantizer input, resulting in $z_{i}$ (see Fig. 1). Accordingly, the quantized and dithered RSS measurement $r_{i}^{d}$ becomes

$$
\begin{aligned}
& r_{i}^{d}=Q\left(z_{i}\right), \\
& z_{i} \triangleq g_{i}(\mathbf{x})+n_{i}+v_{i},
\end{aligned}
$$

We note that the extension of our signal model above to the 3-D case, multiple measurements per anchor, and cooperative paradigm is straightforward.

\section{MAXIMUM LIKELIHOOD ESTIMATION}

The maximum likelihood estimator (MLE) is favorable to use because it is asymptotically efficient [11]. However, for a small number of coarsely quantized observations, the estimator usually suffers from a large bias. It has been studied in [5], [8] that in general, adding dither noise enhances the performance of ML parameter estimation. In the sequel, we borrow this idea to solve the localization problem.

\section{A. MLE without Dithering}

The straightforward solution is to build an MLE directly based on the quantized RSS measurements [12]. For each quantized measurement, say $r_{i}$, it is easy to derive

$$
\begin{aligned}
\operatorname{Pr}\left(r_{i}=s ; \mathbf{x}\right) & =\int_{P_{s}}^{P_{s+1}} p_{y_{i}}(y) \mathrm{d} y \\
& =\Phi\left(\frac{P_{s+1}-g_{i}(\mathbf{x})}{\sigma_{i}}\right)-\Phi\left(\frac{P_{s}-g_{i}(\mathbf{x})}{\sigma_{i}}\right),
\end{aligned}
$$

where $s \in\{1,2, \cdots, S\}$ and $\Phi(\cdot)$ is the standard Gaussian cumulative distribution function (CDF).

Given a set of quantized RSS measurements $r_{1}, r_{2}, \cdots, r_{N}$, the MLE $\hat{\mathbf{x}}_{\mathrm{ML}}$ is given by

$$
\hat{\mathbf{x}}_{\mathrm{ML}}=\arg \max _{\mathbf{x}} \sum_{i=1}^{N} \ln \left(\operatorname{Pr}\left(r_{i}=s ; \mathbf{x}\right)\right) .
$$

\section{B. MLE with Dithering}

The MLE without dithering usually suffers from a relatively large bias, arising from the quantization effect. To motivate this, let us consider the following intuitive example. Suppose we want to quantize a fixed number 0.2 using a 1-bit quantizer with a threshold 0.5 and quantization outputs 0 and 1 . In this case, we will always get 0 and the bias turns out to be 0.2 . If dither noise is added, occasionally the quantizer output will be 1 and the expectation should be closer to 0.2 . Adding a proper dither noise provides the unquantized but dithered signal more diversity and this helps alleviate the undesired quantization effect. Note that, although the dither noise adds uncertainty to the measurements, at least the information is not misinterpreted [8].

Due to the independence between the measurement noise $n_{i}$ and the dither noise $v_{i}$, the probability density function (PDF) of the quantizer input is simply given as

$$
p_{z_{i}}(z)=p_{y_{i}}(z) * p_{v_{i}}(z)
$$

where $*$ represents convolution and $p_{v_{i}}(\cdot)$ denotes the dither noise PDF. Accordingly, we have

$$
\operatorname{Pr}\left(r_{i}^{d}=s ; \mathbf{x}\right)=\int_{P_{s}}^{P_{s+1}} p_{z_{i}}(z) \mathrm{d} z .
$$

The MLE with dithering, $\hat{\mathbf{x}}_{\mathrm{ML}}^{d}$, is given by

$$
\hat{\mathbf{x}}_{\mathrm{ML}}^{d}=\arg \max _{\mathbf{x}} \sum_{i=1}^{N} \ln \left(\operatorname{Pr}\left(r_{i}^{d}=s ; \mathbf{x}\right)\right) .
$$

Selecting an appropriate dither noise distribution is vital. As stated in [9], a Gaussian distribution with standard deviation $\sigma_{v}$ fulfilling $\sigma_{v} / \Delta \geq 0.5$ ( $\Delta$ is the quantization step-size) satisfies almost perfectly quantization theorem I in [9], under which full reconstruction of the unquantized but dithered signal is possible. Another type of dither noise that fulfills quantization theorem I in [9] rigorously, has been proposed in [8]. Both types of dither noise are considered in this paper. Motivated by the satisfactory performance of the uniform dithering in [6], uniformly distributed dither noise is considered as well. For each dither noise type, we derive the corresponding likelihood function in the sequel. For notational brevity, we will ignore the subscript $i$.

1) Gaussian Distribution: Suppose the dither noise $v$ is a zero-mean Gaussian random variable with variance $\sigma_{v}^{2}$. It is easy to prove that the PDF in Eq. (1) can be expressed as,

$$
p_{z}(z)=\frac{1}{\sqrt{2 \pi} \sigma_{d}} \exp \left\{-\frac{(z-g(\mathbf{x}))^{2}}{2 \sigma_{d}^{2}}\right\},
$$

where $\sigma_{d}^{2} \triangleq \sigma^{2}+\sigma_{v}^{2}$. Accordingly, this results in a closed-form probability according to Eq. (2)

$$
\operatorname{Pr}\left(r^{d}=s ; \mathbf{x}\right)=\Phi\left(\frac{P_{s+1}-g(\mathbf{x})}{\sigma_{d}}\right)-\Phi\left(\frac{P_{s}-g(\mathbf{x})}{\sigma_{d}}\right) .
$$

2) Distribution of $\operatorname{Sinc}^{2 \alpha}(\cdot)$ Form: The dither noise distribution proposed in [8] is given by

$$
p_{v}(v)=\frac{1}{c} \operatorname{sinc}^{2 \alpha}\left(\frac{\pi v}{2 \alpha \Delta_{d}}\right)
$$

where $c$ is a normalization factor, $\alpha$ is an integer commonly ranging from 1 to 3 , and $\Delta_{d}$ is a key parameter that controls the dispersion of the distribution. For a strict satisfaction of the quantization theorem I in [9], we need to choose $\Delta_{d}=\Delta$. In the sequel, we will refer to this distribution as the $\operatorname{sinc}^{2 \alpha}(\cdot)$ distribution. Generation of $\operatorname{sinc}^{2 \alpha}(\cdot)$ distributed dither noise 


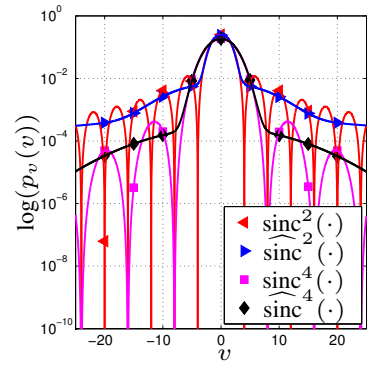

Fig. 2: Comparison of the $\operatorname{sinc}^{2 \alpha}(\cdot)$ distribution and a Gaussian mixture approximation with 8 and 4 components for $\alpha=1$ and 2 , respectively.

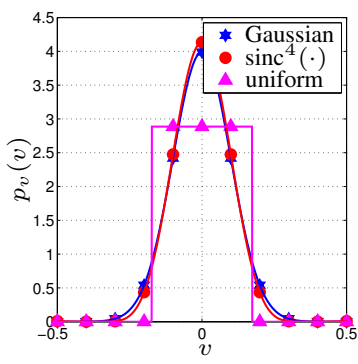

Fig. 3: Different dither noise distributions having the same standard deviation $\sigma_{v}=0.1 \Delta$, and with quantization step-size $\Delta=1$.

was introduced in Algorithm 3 in [8]. Unfortunately, a closedform expression of the likelihood function may not exist. We propose to approximate the $\operatorname{sinc}^{2 \alpha}(\cdot)$ distribution using a Gaussian mixture model (GMM) given by

$$
p_{v}(v) \approx \sum_{l=1}^{L} w_{l} p_{\mathcal{N}}\left(v ; \mu_{l}, \sigma_{l}^{2}\right)
$$

where $L$ is the number of Gaussian components, $w_{l}$ are the mixing components normalized to sum up to 1 , and $p_{\mathcal{N}}\left(v ; \mu_{l}, \sigma_{l}^{2}\right)$ denotes the Gaussian PDF with mean $\mu_{l}$ and variance $\sigma_{l}^{2}$. Using the GMM approximation yields

$$
\begin{aligned}
& \operatorname{Pr}\left(r^{d}=s ; \mathbf{x}\right) \approx \\
& \sum_{l=1}^{L} w_{l}\left(\Phi\left(\frac{P_{s+1}-\mu_{l}-g(\mathbf{x})}{\sqrt{\sigma^{2}+\sigma_{l}^{2}}}\right)-\Phi\left(\frac{P_{s}-\mu_{l}-g(\mathbf{x})}{\sqrt{\sigma^{2}+\sigma_{l}^{2}}}\right)\right) .
\end{aligned}
$$

The unknown GMM parameters $\left\{w_{l}, \mu_{l}, \sigma_{l}^{2}\right\}_{l=1}^{L}$ are learned offline using the expectation maximization (EM) algorithm [13]. Note that the dither noise is still generated according to the original $\operatorname{sinc}^{2 \alpha}(\cdot)$ distribution given in Eq. (3). The suitability of the GMM for the representation of the $\operatorname{sinc}^{2 \alpha}(\cdot)$ is shown in Fig. 2, where $\widehat{\operatorname{sinc}}^{2 \alpha}(\cdot)$ in the legend denotes the approximated $\operatorname{sinc}^{2 \alpha}(\cdot)$ using the GMM. Note that, even though we found that actually one Gaussian distribution captures the $\operatorname{sinc}^{4}(\cdot)$ distribution very well, four Gaussians are used for enhanced accuracy.

3) Uniform Distribution: Consider a uniformly distributed dither noise

$$
p_{v}(v)=\left\{\begin{array}{cl}
1 / \Delta_{u}, & \text { if }-\Delta_{u} / 2 \leq v<\Delta_{u} / 2 \\
0, & \text { otherwise }
\end{array}\right.
$$

where $\Delta_{u}$ is a parameter which defines the support of the uniform distribution. Using Eq. (4), the PDF in Eq. (1) can be expressed as

$p_{z}(z)=\frac{1}{\Delta_{u}}\left(\Phi\left(\frac{z+\frac{\Delta_{u}}{2}-g(\mathbf{x})}{\sigma}\right)-\Phi\left(\frac{z-\frac{\Delta_{u}}{2}-g(\mathbf{x})}{\sigma}\right)\right)$.

\begin{tabular}{c||c|c|c|c|c|c|c}
\hline Parameter & $A_{0}$ & $n_{p}$ & $\sigma_{i}^{2}$ & $d_{0}$ & $\alpha$ & $L$ & $\Delta(S=2)$ \\
\hline Value & -50 & 2.3 & 16 & 1 & 2 & 4 & 25 \\
\hline
\end{tabular}

TABLE I: Simulation parameters

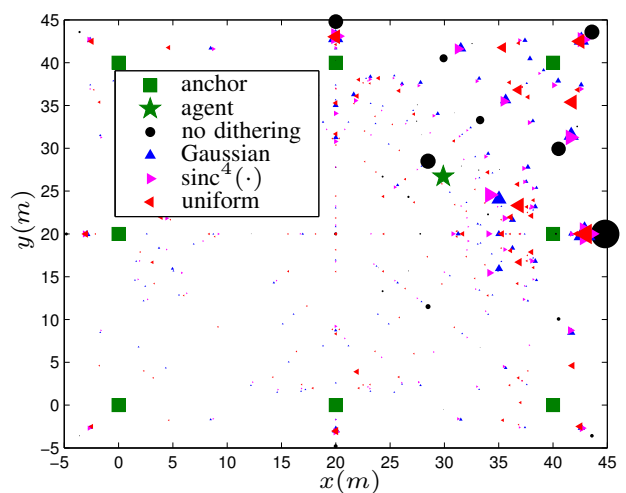

Fig. 4: Location estimates based on 1-bit quantized RSS measurements.

For uniform dither noise, the probability in Eq. (2) is available in closed form and is given by

$\operatorname{Pr}\left(r^{d}=s ; \mathbf{x}\right)=$

$\frac{1}{\Delta_{u}}\left(h\left(P_{s}-g(\mathbf{x})-\Delta_{u} / 2\right)-h\left(P_{s}-g(\mathbf{x})+\Delta_{u} / 2\right)\right.$

$\left.-h\left(P_{s+1}-g(\mathbf{x})-\Delta_{u} / 2\right)+h\left(P_{s+1}-g(\mathbf{x})+\Delta_{u} / 2\right)\right)$

$+\frac{\sigma^{2}}{\Delta_{u}}\left(p_{\mathcal{N}}\left(P_{s} ; g(\mathbf{x})+\Delta_{u} / 2, \sigma^{2}\right)-p_{\mathcal{N}}\left(P_{s} ; g(\mathbf{x})-\Delta_{u} / 2, \sigma^{2}\right)\right.$

$\left.-p_{\mathcal{N}}\left(P_{s+1} ; g(\mathbf{x})+\Delta_{u} / 2, \sigma^{2}\right)+p_{\mathcal{N}}\left(P_{s+1} ; g(\mathbf{x})-\Delta_{u} / 2, \sigma^{2}\right)\right)$,

where $h(m) \triangleq m \cdot \Phi\left(\frac{m}{\sigma}\right)$.

\section{Simulation Results}

In this section, we evaluate all MLEs in terms of bias and root-mean-square-error (RMSE). The bias is redefined as the average of the absolute bias in $x$ and $y$-direction. A sensor network over an area of $40 \times 40 \mathrm{~m}$ is used, where 8 anchors locate at the four corners and the center positions along four borders. The agent position locates approximately at $[29.9,26.7]^{T}$, and 5000 independent Monte Carlo runs have been used to generate the measurements. The quantizer thresholds $\left\{P_{2}, \cdots, P_{S}\right\}$ are chosen such that they equally partition the range $[-100,-50]$ $\mathrm{dBm}$ into $S$ regions. An overview of the simulation parameters is given in Table I. The estimates are found numerically by using the fminsearch function in MATLAB. The initial value is set to $\mathbf{x}$ plus a vector uniformly distributed in the range $[-2.5,2.5]$.

In the first simulation, we set the variance of different dither noise distributions to be the same and only focus on the influence of their PDF shapes. An example of three dither noise PDFs with the same variance is plotted in Fig. 3. As the $\operatorname{sinc}^{2}(\cdot)$ distribution has infinite variance, we choose $\operatorname{sinc}^{4}(\cdot)$ as a representative for the $\operatorname{sinc}^{2 \alpha}(\cdot)$ family. We conservatively start with a small dither noise by setting $\sigma_{v}$ to $0.2 \Delta$, since a large dither noise will probably lead to a large increase in the estimation variance and RMSE. For $S=2$, the position estimates of all MLEs are depicted in Fig. 4, where the size 

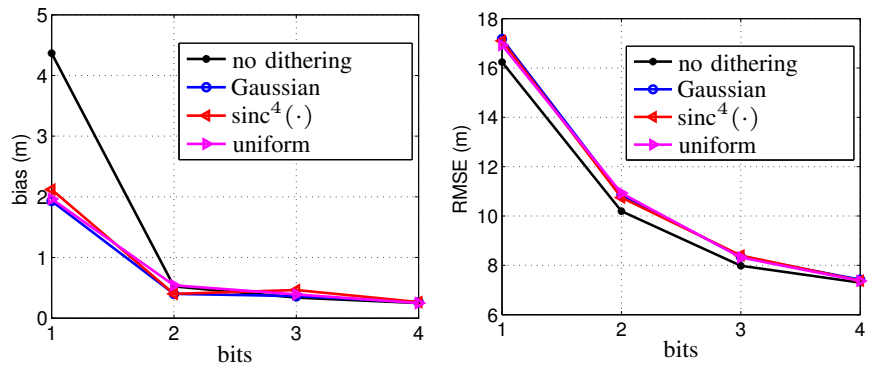

Fig. 5: Localization performance vs. number of quantization bits

of the marker is proportional to the frequency of occurrence of an estimate at that location. It is obvious that in most cases the MLE without dithering gives rise to an estimate that is far away from the true agent position. However, the position estimates obtained by the MLEs with dithering are comparatively closer to the true position. Furthermore, the bias and RMSE of all MLEs are evaluated over the number of bits required for the quantization $\left(S=2^{\text {bits }}\right)$ and the results are shown in Fig. 5. Three main results are obtained. First, as the quantization becomes finer, as expected, all MLEs deliver more accurate estimation results. Second, for all three dither noise distributions, the bias of the location estimator is reduced as compared to the MLE without dithering, but, at a cost of a slightly increased RMSE. Third, as the number of bits adopted for quantization increases, the benefit of the MLEs using dithering diminishes, i.e., both MLEs with and without dithering demonstrate comparable performance in bias.

To gain a comprehensive understanding about dithering, we further evaluate the influence of the variance of dither noise on the performance. In the second simulation, only 1-bit quantization is considered. The evaluation results are shown in Fig. 6, where $\sigma_{v} / \Delta$ denotes the ratio of the standard deviation of the dither noise and the quantization step-size. As shown, the bias is substantially decreased by using dithering. As the variance of the dither noise increases, the estimation bias first decreases and then turns to increase. This is logical, since an appropriately chosen dither noise gives the unquantized signal enough diversity to reduce the estimation bias. However, since dither noise introduces additional uncertainty, a too large chosen dither noise will deteriorate the performance. For the MLEs with dithering, the RMSE increases with the variance of the dither noise. A good balance is achieved by the dither noise of relatively small variance, such as $0.2 \leq \sigma_{v} / \Delta \leq 0.3$. The choice of the dither noise distribution and whether it fulfills quantization theorem I or not seems to have only a minor effect on the estimation performance, since all MLEs under investigation have comparable performance for the chosen simulation scenario.

It can be concluded, that for 1-bit quantized measurements, it is beneficial to adopt the MLE with dithering. The choice of a specific dither noise PDF seems to play only a minor role. In order to achieve a reduction in bias and preserve a comparable RMSE, we suggest to adopt a dither noise with relatively small variance.

\section{Conclusion}

In this paper, we have addressed the problem of localization based on quantized RSS measurements. To alleviate the
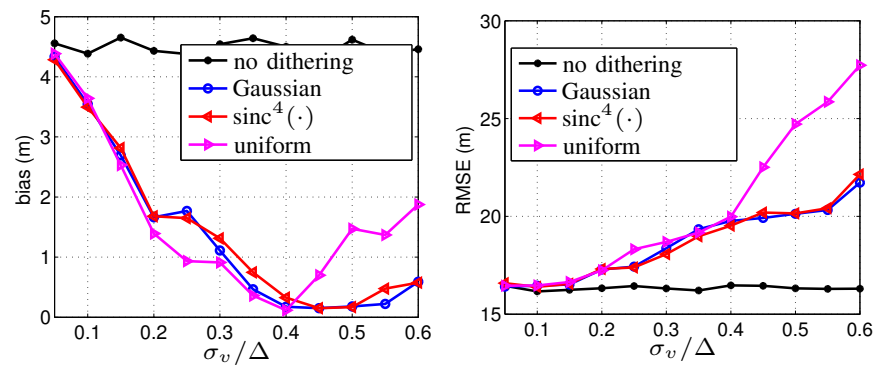

Fig. 6: Localization performance vs. dither noise variance

quantization effect in the measurements, we have proposed a maximum-likelihood estimator with dithering. A rather thorough investigation on different dither noise distributions has been conducted and the corresponding likelihood functions have been derived. Our simulation results have demonstrated that dithering is beneficial for coarsely quantized RSS measurements in terms of reduced bias. A shortcoming of the proposed MLE with dithering is that dithering lead to an increase in the estimation variance.

\section{REFERENCES}

[1] R. Zekavat and R. M. Buehrer, Handbook of Position Location. Hoboken, NJ: John Wiley \& Sons, Inc., 2011.

[2] Y. Zhao, F. Yin, and F. Gunnarsson, "Particle filtering for positioning based on proximity reports," in Proc. Int. Conf. on Information Fusion, Washington, D.C., USA, July 2015.

[3] H. Papadopoulos, G. W. Wornell, and A. Oppenheim, "Sequential signal encoding from noisy measurements using quantizers with dynamic bias control," IEEE Trans. Inf. Theory, vol. 47, no. 3, pp. 978-1002, Mar. 2001.

[4] A. Ribeiro and G. B. Giannakis, "Bandwidth-constrained distributed estimation for wireless sensor networks-part II: Unknown PDF," IEEE Trans. on Signal Process., vol. 54, no. 7, pp. 2784-2796, Jul. 2006.

[5] F. Gustafsson and R. Karlsson, "Statistical results for system identification based on quantized observations," Automatica, vol. 45, no. 12, pp. 2794-2801, Dec. 2009.

[6] O. Dabeer and A. Karnik, "Signal parameter estimation using 1-bit dithered quantization," IEEE Trans. Inf. Theory, vol. 52, no. 12, pp. 5389-5405, Dec. 2006.

[7] O. Dabeer and E. Masry, "Multivariate signal parameter estimation under dependent noise from 1-bit dithered quantized data," IEEE Trans. Inf. Theory, vol. 54, no. 4, pp. 1637-1654, Apr. 2008.

[8] F. Gustafsson and R. Karlsson, "Generating dithering noise for maximum likelihood estimation from quantized data," Automatica, vol. 49, no. 2, pp. 554-560, Feb. 2013.

[9] B. Widrow and I. Kollár, Quantization Noise: Roundoff Error in Digital Computation, Signal Processing, Control, and Communications. Cambridge, UK: Cambridge University Press, 2008.

[10] F. Gustafsson, "Particle filter theory and practice with positioning applications," IEEE Aerospace and Electronic Systems Magazine, vol. 25, no. 7, pp. 53-82, July 2010.

[11] S. M. Kay, Fundamentals of Statistical Signal Processing: Estimation Theory. Upper Saddle River, NJ, USA: Prentice-Hall, 1993.

[12] N. Patwari and A. O. Hero, III, "Using proximity and quantized RSS for sensor localization in wireless networks," in Proceedings of the 2nd ACM International Conference on Wireless Sensor Networks and Applications, New York, NY, USA, 2003, pp. 20-29.

[13] A. P. Dempster, N. M. Laird, and D. B. Rubin, "Maximum likelihood from incomplete data via the EM algorithm," Journal of the Royal Statistical Society, Series B, vol. 39, no. 1, pp. 1-38, 1977. 